Article

\title{
Iron(III)-Catalyzed Highly Regioselective Halogenation of 8-Amidoquinolines in Water
}

\author{
Yang Long ${ }^{\dagger}$, Lei Pan ${ }^{\dagger}$ and Xiangge Zhou * \\ College of Chemistry, Sichuan University, Chengdu 610064, China; elitelyhere@163.com (Y.L.); \\ panleiscu@163.com (L.P.) \\ * Correspondence: zhouxiangge@scu.edu.cn; Tel.: +86-28-85412904 \\ + These authors contributed equally to this work.
}

Academic Editor: Kei Saito

Received: 27 December 2018; Accepted: 31 January 2019; Published: 1 February 2019

check for updates

Abstract: A simple protocol of iron(III)-catalyzed halogenation of 8-amidoquinolines in water under mild conditions was developed, affording the 5-halogenlated products in good to excellent yields up to $98 \%$. The reaction mechanism most likely involves a single-electron transfer (SET) process.

Keywords: 8-amidoquinoline; iron; catalysis; water

\section{Introduction}

Quinolines are an important kind of structural motif found in numerous bioactive molecules and natural products with applications in pharmaceutical chemistry. Over the past decades, the quinoline framework has drawn significant attention due to its frequent occurrence in bioactive natural products, agrochemicals, and functional materials (Scheme 1) [1-8]. In addition, with the seminal achievement of using 8-amidoquinoline as a bidentate directing group by Daugulis [9], a great deal of C-H functionalization reaction has been realized [10]. Therefore, the development of facile and efficient methods to halogenate quinolines is highly necessary. However, compared with well-developed functionalization of quinoline's C2, C3, and C8 positions [11-19], the approaches to the C5-functionalization are still less studied.

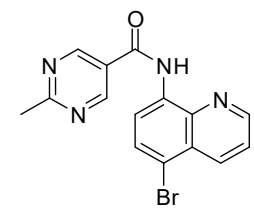

Antitumor agents

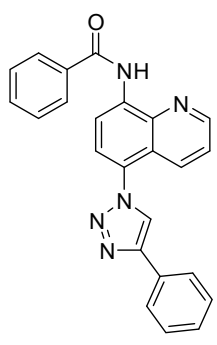

Antimalarials<smiles>CN(C)CCNCc1ccc2c(Cl)c(Cl)cc(N)c2n1</smiles>

Nervous system agents

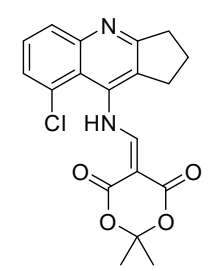

Anti-Alzheimer agents

Scheme 1. Examples of biologically active compounds containing quinoline motifs.

Recently, Stahl and co-workers reported $\mathrm{Cu}(\mathrm{I})$ catalyzed $\mathrm{C} 5$ chlorination of 8-amidoquinoline amides by using $\mathrm{LiCl}$ as a chlorination reagent [20]. Similarly, Zhang, Li, Huang, etc. described 
copper-catalyzed C5 C-H halogenation of 8-amidoquinolines (Scheme 2a) [21-27]. Meanwhile, $\mathrm{Li}$ and other groups also reported transition metal-free $\mathrm{C} 5$ halogenation of 8-amidoquinolines (Scheme 2b) [28-31]. Although approaches to the C5 halogenation have been developed to a certain extent, the work mentioned above still has some drawbacks: (i) usually there was a requirement of some complex oxidants, such as $\mathrm{N}$-fluorobis(benzenesulfon)imide (NFSI), $\mathrm{PhI}(\mathrm{OAc})_{2}$, oxone, etc.; (ii) the halogen reagents were expensive, low cost resources like $\mathrm{Br}_{2}$ or $\mathrm{I}_{2}$ that hardly reacted effectively in these systems [25]; (iii) normally there was usage of an organic solvent, while using water as the sole solvent was pretty rare [32]; (iv) some reactions needed harsh conditions, such as high temperature or inert atmosphere. In continuation of our recent work of aqueous catalysis [33-40], herein is reported iron-catalyzed remote $\mathrm{C} 5 \mathrm{C}-\mathrm{H}$-halogenation of 8 -amidoquinolines in water by using NXS and $\mathrm{X}_{2}$ $(\mathrm{X}=\mathrm{Br}, \mathrm{I})$ ) as effective halogen sources (Scheme 2c). Notably, we think this protocol is environmentally friendly with the following novelties: (i) water as a solvent and air as an oxidant; (ii) cheap iron salt as the catalyst at mild reaction conditions at room temperature [41]; (iii) easily available and budget-friendly halogen reagents.

\section{Previous work}

(a)
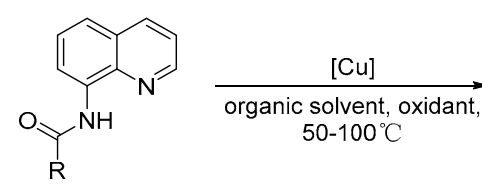

Halogen reagents: $\mathrm{NaX}, \mathrm{LiCl}, \mathrm{CuCl}_{2}$ Oxidant: NFSI, $\mathrm{Phl}(\mathrm{OAc})_{2}$, etc (ref. 18-25) (b)

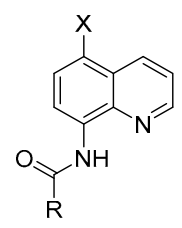<smiles>[R]C(=O)Nc1cccc2cccnc12</smiles>

transition metal-free $\overrightarrow{\text { organic solvent, oxidant, }}$ $\mathrm{rt}-100^{\circ} \mathrm{C}$<smiles>[R]C(=O)NC</smiles>

Halogen reagents: NaX, NBS, TBCA Oxidant: oxone, $\mathrm{K}_{2} \mathrm{~S}_{2} \mathrm{O}_{8}$ (ref. 26-29)

\section{This work}

(c)

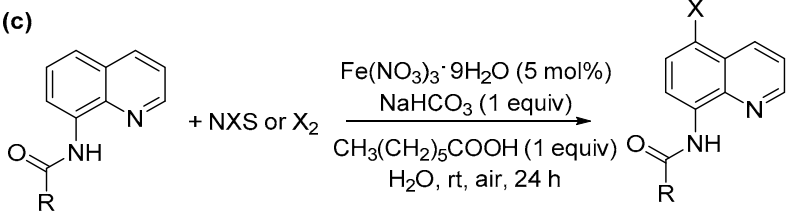

Scheme 2. C5 halogenation of 8-amidoquinoline amides. (a) Cu-catalyzed halogenation of 8-amidoquinoline amides; (b) transition metal-free halogenation of 8-amidoquinoline amides; (c) Fe(III)-catalyzed halogenation of 8-amidoquinoline amides.

\section{Results and Discussion}

Initially, $\mathrm{N}$-(quinolin-8-yl)pivalamide, $\mathrm{N}$-bromo-succinimide (NBS) or $\mathrm{Br}_{2}$ were treated as model substrates to optimize the reaction conditions. As shown in Table 1, no desired product was observed under an inert atmosphere (entry 1), indicating the indispensability of the oxidant. Furthermore, although the product could be obtained with 91\% yield by NBS without a catalyst, the yield was only $35 \%$ with $\mathrm{Br}_{2}$ as a halogen source (entry 2). Thus, in order to make both halogen reagents react well, a variety of metal salts including $\mathrm{Pd}(\mathrm{II}), \mathrm{Cu}(\mathrm{II}), \mathrm{Co}(\mathrm{II})$, and $\mathrm{Fe}(\mathrm{III})$ were then evaluated. To our delight, metal salts were helpful for the catalysis, especially in the case of $\mathrm{Br}_{2}$ as a halogen source, the reactivity of which was improved to the same level as NBS (entries 3-7). Considering the efficiency, low cost, and environmental friendliness, $\mathrm{Fe}\left(\mathrm{NO}_{3}\right)_{3} \cdot 9 \mathrm{H}_{2} \mathrm{O}$ was selected as a catalyst for the further studies. The oxidant was another important factor that affected the results. Simple silver salts such as $\mathrm{Ag}_{2} \mathrm{O}$ and $\mathrm{Ag}_{2} \mathrm{CO}_{3}$ gave similar yields as air (entries 8 and 9), while $\mathrm{AgOAc}$ raised the yield to about $75 \%$ (entry 10). Interestingly, the yield increased dramatically to about $90 \%$ in the case of $\mathrm{CH}_{3}\left(\mathrm{CH}_{2}\right)_{5} \mathrm{COOAg}$ (entry 11), indicating the positive effect of a long chain carboxylic acid ion, which might function as a phase transfer reagent [42]. Indeed, when the air was used as an oxidant, the combination of $\mathrm{CH}_{3}\left(\mathrm{CH}_{2}\right)_{5} \mathrm{COOH}$ and $\mathrm{NaHCO}_{3}$ resulted in the best yield of $95 \%$ for both halogen 
reagents (entry 12). In summary, the optimal conditions consist of quinolines $(0.3 \mathrm{mmol})$, $\mathrm{NBS}$ or $\mathrm{Br}_{2}$ $(0.6 \mathrm{mmol}), \mathrm{Fe}\left(\mathrm{NO}_{3}\right)_{3} \cdot 9 \mathrm{H}_{2} \mathrm{O}(5 \mathrm{~mol} \%), \mathrm{NaHCO}_{3}(0.3 \mathrm{mmol})$, and $\mathrm{CH}_{3}\left(\mathrm{CH}_{2}\right)_{5} \mathrm{COOH}(0.3 \mathrm{mmol})$ at room temperature for $24 \mathrm{~h}$ in the air.

Table 1. Optimization of the reaction conditions. ${ }^{\text {a }}$

\begin{tabular}{|c|c|c|c|c|}
\hline \multirow{2}{*}{ Entry } & \multirow{2}{*}{ Catalyst (\%) } & \multirow{2}{*}{ Additive } & \multicolumn{2}{|c|}{ Yield $[\%]^{b}$} \\
\hline & & & Using $\mathrm{Br}_{2}$ & Using NBS \\
\hline $1^{\mathrm{c}}$ & - & - & 0 & 0 \\
\hline 2 & - & - & 35 & 91 \\
\hline 3 & $\mathrm{Pd}(\mathrm{OAc})_{2}(10)$ & - & 47 & 67 \\
\hline 4 & $\mathrm{Cu}(\mathrm{OAc})_{2}(20)$ & - & 43 & 65 \\
\hline 5 & $\mathrm{Co}(\mathrm{OAc})_{2} \cdot 9 \mathrm{H}_{2} \mathrm{O}(20)$ & - & 39 & 58 \\
\hline 6 & $\mathrm{Fe}\left(\mathrm{NO}_{3}\right)_{3} \cdot 9 \mathrm{H}_{2} \mathrm{O}(5)$ & - & 68 & 68 \\
\hline 7 & $\mathrm{FeCl}_{3} \cdot 9 \mathrm{H}_{2} \mathrm{O}(5)$ & - & 63 & 65 \\
\hline 8 & $\mathrm{Fe}\left(\mathrm{NO}_{3}\right)_{3} \cdot 9 \mathrm{H}_{2} \mathrm{O}(5)$ & $\mathrm{Ag}_{2} \mathrm{O}$ & 59 & 65 \\
\hline 9 & $\mathrm{Fe}\left(\mathrm{NO}_{3}\right)_{3} \cdot 9 \mathrm{H}_{2} \mathrm{O}(5)$ & $\mathrm{Ag}_{2} \mathrm{CO}_{3}$ & 64 & 69 \\
\hline 10 & $\mathrm{Fe}\left(\mathrm{NO}_{3}\right)_{3} \cdot 9 \mathrm{H}_{2} \mathrm{O}(5)$ & AgOAc & 73 & 75 \\
\hline 11 & $\mathrm{Fe}\left(\mathrm{NO}_{3}\right)_{3} \cdot 9 \mathrm{H}_{2} \mathrm{O}(5)$ & $\mathrm{CH}_{3}\left(\mathrm{CH}_{2}\right)_{5} \mathrm{COOAg}$ & 89 & 90 \\
\hline 12 & $\mathrm{Fe}\left(\mathrm{NO}_{3}\right)_{3} \cdot 9 \mathrm{H}_{2} \mathrm{O}(5)$ & $\begin{array}{c}\mathrm{CH}_{3}\left(\mathrm{CH}_{2}\right)_{5} \mathrm{COOH} \\
\mathrm{NaHCO}_{3} \mathrm{~d}\end{array}$ & 95 & 95 \\
\hline
\end{tabular}

a Unless otherwise noted, the reactions were carried out with $N$-(quinolin-8-yl)pivalamide $(0.3 \mathrm{mmol})$, $\mathrm{N}$-bromo-succinimide (NBS) or $\mathrm{Br}_{2}(0.6 \mathrm{mmol})$, a catalyst $(5-20 \%)$, an additive $(0.6 \mathrm{mmol})$ in water $(1 \mathrm{~mL})$ under air atmosphere at room temperature for $24 \mathrm{~h} .{ }^{\mathrm{b}}$ Isolated yields. ${ }^{\mathrm{c}} \mathrm{N}_{2}$ atmosphere. ${ }^{\mathrm{d}} \mathrm{CH}_{3}\left(\mathrm{CH}_{2}\right)_{5} \mathrm{COOH}(0.3 \mathrm{mmol})$, $\mathrm{NaHCO}_{3}(0.3 \mathrm{mmol})$.

With the optimized conditions in hand, we subsequently examined the scope of quinoline derivatives, as shown in Scheme 3. Overall, different substrates provided moderate to excellent yields, and both halogen reagents could efficiently realize the reaction, while much lower reactive activity was found in the previous reports [26]. The length of the linear alkyl chain showed few effects on the reaction, affording similar results around $90 \%$ yields (Scheme $3 a-d$ ). Various branched chain alkyl groups also gave excellent yield (Scheme $3 \mathrm{e}-\mathrm{j}$ ). Meanwhile, different aryl groups were also compatible in this system. The substrates bearing para-methyl chloro groups gave excellent yields up to $95 \%$ (Scheme 31,m), para-trifluoromethyl groups gave a moderate yield of about $73 \%$ (Scheme 3n), and the meta-chloro and methoxyl group gave a good yield (Scheme 3o,p). Replacement of the aryl with the ethylphenyl and thienyl groups were also well-tolerated (Scheme 3q,r). The methyl group on the $\mathrm{C} 2$ position of the quinoline ring were also compatible (Scheme 3s). Moreover, the structure of the product (Scheme 31) was confirmed by X-ray crystallography (Figure 1) [CCDC 1480727, for detailed crystal data, see Supplementary Information (SI)].

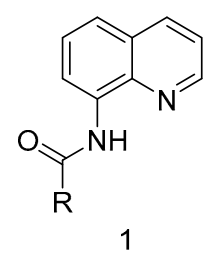

1

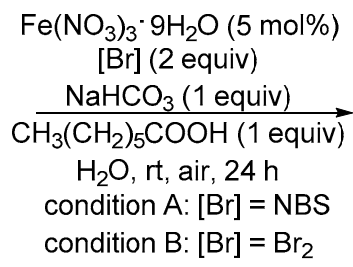<smiles>[R]C(=O)Nc1ccc(Br)c2cccnc12</smiles> 


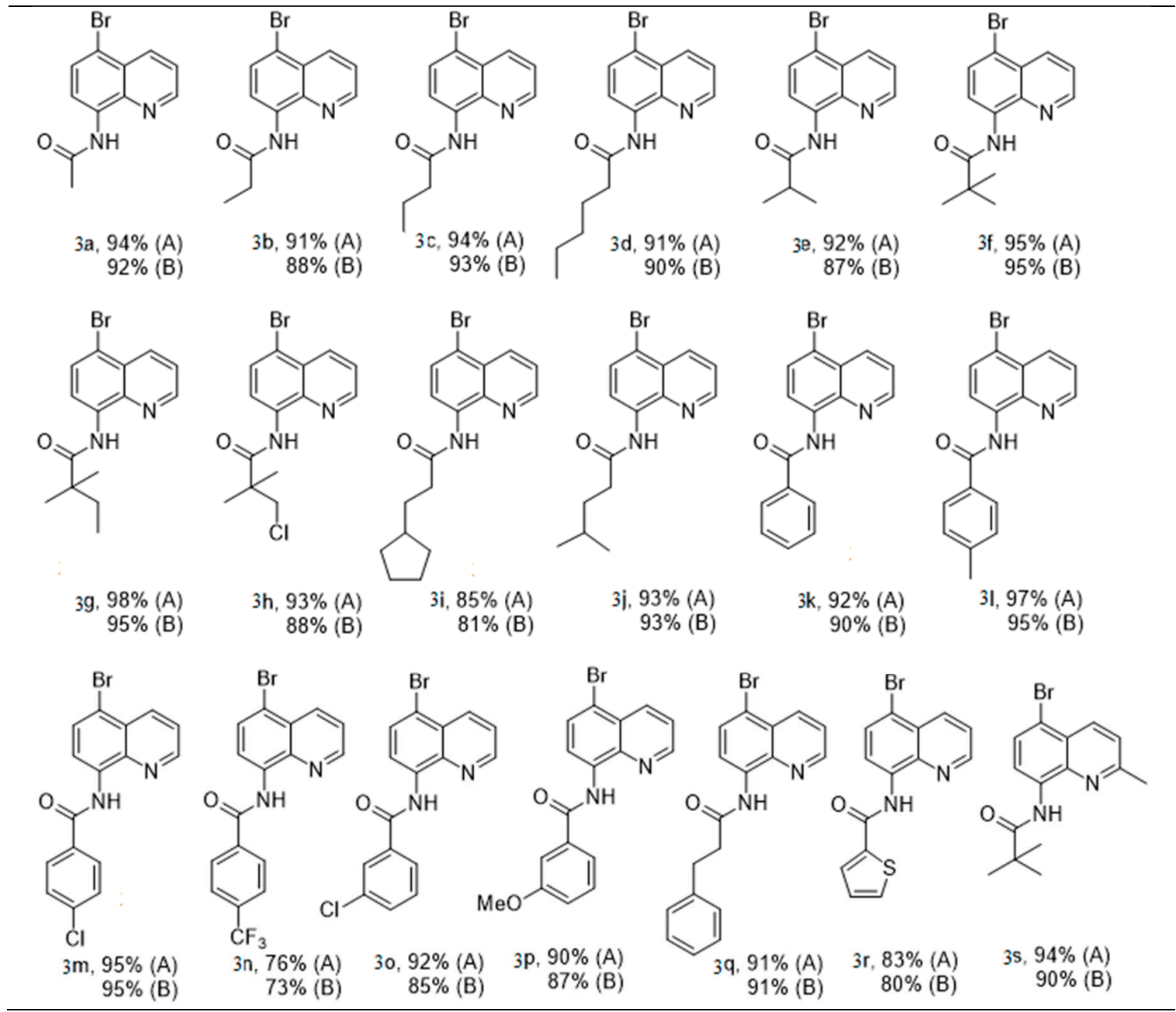

Scheme 3. Iron-catalyzed C-H bromination. Reaction conditions A: $1(0.3 \mathrm{mmol})$, NBS (0.6 mmol), $\mathrm{CH}_{3}\left(\mathrm{CH}_{2}\right)_{5} \mathrm{COOH}(0.3 \mathrm{mmol}), \mathrm{NaHCO}_{3}(0.3 \mathrm{mmol})$, and $\mathrm{Fe}\left(\mathrm{NO}_{3}\right)_{3} \cdot 9 \mathrm{H}_{2} \mathrm{O}(5 \mathrm{~mol} \%)$ in water $(1 \mathrm{~mL})$ at $\mathrm{rt}$ for $24 \mathrm{~h}$. Reaction conditions B: 1 (0.3 mmol), $\mathrm{Br}_{2}(0.6 \mathrm{mmol}), \mathrm{CH}_{3}\left(\mathrm{CH}_{2}\right)_{5} \mathrm{COOH}(0.3 \mathrm{mmol}), \mathrm{NaHCO}_{3}$ $(0.3 \mathrm{mmol})$, and $\mathrm{Fe}\left(\mathrm{NO}_{3}\right)_{3} \cdot 9 \mathrm{H}_{2} \mathrm{O}(5 \mathrm{~mol} \%)$ in water $(1 \mathrm{~mL})$ at $\mathrm{rt}$ for $24 \mathrm{~h}$.

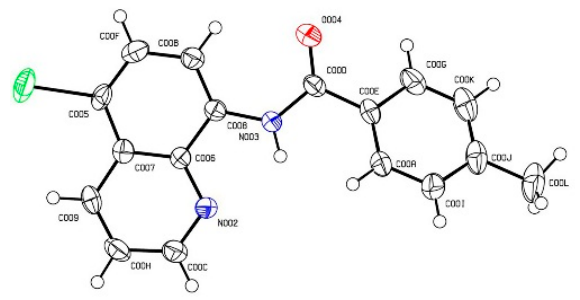

Figure 1. Single-crystal X-ray structure of 21.

In an endeavor to expand the scope of this methodology, NIS and $\mathrm{I}_{2}$ were treated as halogen reagents. As shown in Scheme 4, iodination reaction could also be fulfilled (although with low yields around $40 \%$ ) by using $\mathrm{I}_{2}$ or NIS (3c, 3f). The lower yield of iodination than that of bromination might have been due to the lower reactivity of the iodine free radical and the instability of iodo products $[43,44]$. 

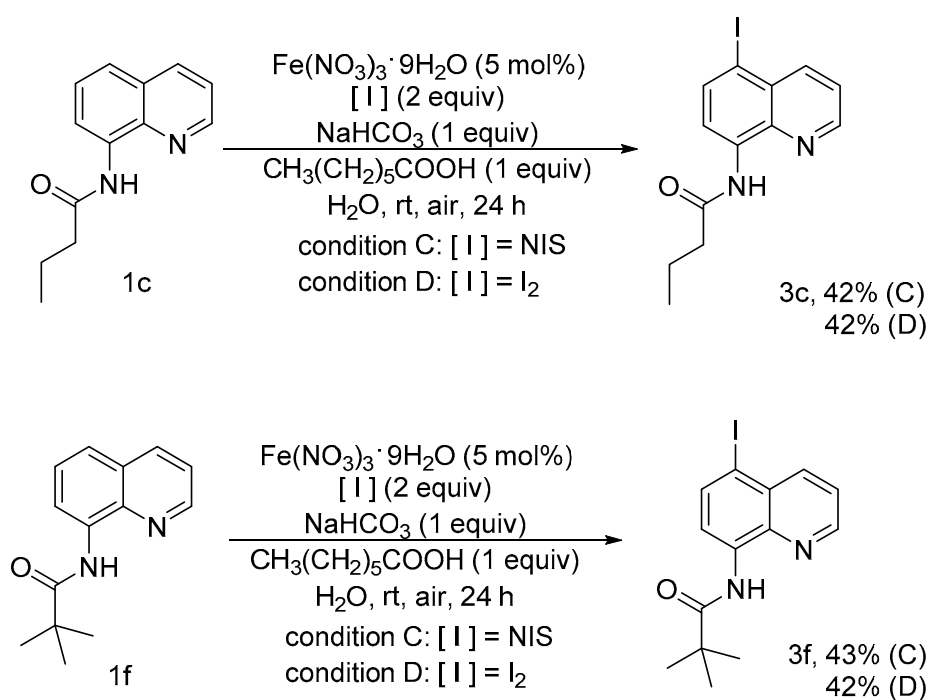

Scheme 4. Iron-catalyzed C-H halogenation. Reaction conditions C: 1 (0.3 mmol), NIS (0.6 mmol), $\mathrm{CH}_{3}\left(\mathrm{CH}_{2}\right)_{5} \mathrm{COOH}(0.3 \mathrm{mmol}), \mathrm{NaHCO}_{3}(0.3 \mathrm{mmol})$, and $\mathrm{Fe}\left(\mathrm{NO}_{3}\right)_{3} \cdot 9 \mathrm{H}_{2} \mathrm{O}(5 \mathrm{~mol} \%)$ in water $(1 \mathrm{~mL})$ at rt for $24 \mathrm{~h}$. Reaction conditions D: $1(0.3 \mathrm{mmol}), \mathrm{I}_{2}(0.6 \mathrm{mmol}), \mathrm{CH}_{3}\left(\mathrm{CH}_{2}\right)_{5} \mathrm{COOH}(0.3 \mathrm{mmol}), \mathrm{NaHCO}_{3}$ $(0.3 \mathrm{mmol})$, and $\mathrm{Fe}\left(\mathrm{NO}_{3}\right)_{3} \cdot 9 \mathrm{H}_{2} \mathrm{O}(5 \mathrm{~mol} \%)$ in water $(1 \mathrm{~mL})$ at $\mathrm{rt}$ for $24 \mathrm{~h}$.

Furthermore, the scaled-up reaction was carried out, giving quantities of the 2c in 90\% (Scheme 5), which indicated it as a facile route to the desired product on a more synthetically useful scale.<smiles>CCCC(=O)Nc1cccc2cccnc12</smiles>

$5.0 \mathrm{mmol}, 1.07 \mathrm{~g}$<smiles>O=C1CCC(=O)N1Br</smiles>

2 equiv, $1.78 \mathrm{~g}$
$\mathrm{Fe}\left(\mathrm{NO}_{3}\right)_{3} \cdot 9 \mathrm{H}_{2} \mathrm{O}(5 \mathrm{~mol} \%)$ $\mathrm{NaHCO}_{3}$ (1 equiv) $\mathrm{CH}_{3}\left(\mathrm{CH}_{2}\right)_{5} \mathrm{COOH}$ (1 equiv) $\mathrm{H}_{2} \mathrm{O}$, rt, air, $24 \mathrm{~h}$<smiles>CCCC(=O)Nc1ccc(Br)c2cccnc12</smiles>

Yield: $90 \%, 1.31 \mathrm{~g}$

Scheme 5. Gram-scale iron-catalyzed C-H bromination of 1c.

In order to expand the application of this protocol, $N$-(5-bromoquinolin-8-yl)pivalamide was reacted with boronic acid to give a series of derivatives by simple Suzuki coupling reactions in moderate to good yields ranging from $54 \%$ to $84 \%$ (Scheme 6 ) [45,46].

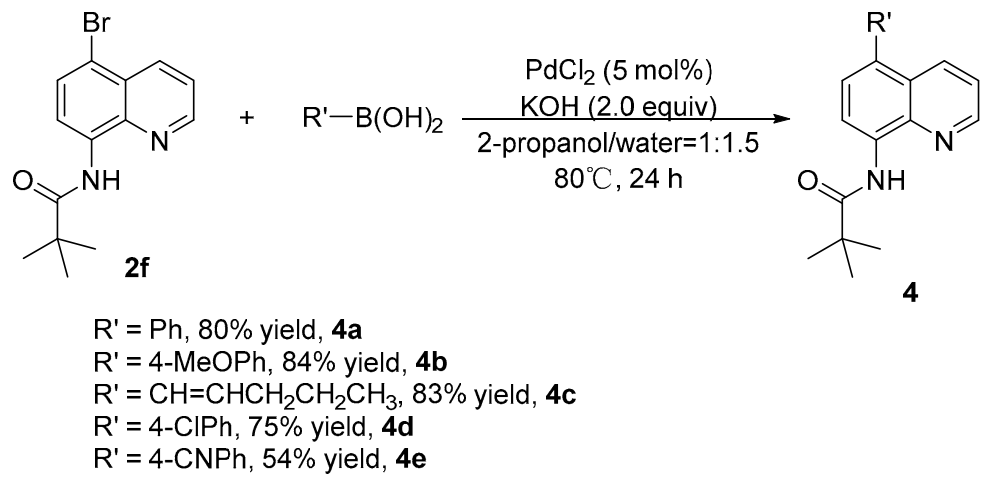

Scheme 6. Suzuki coupling reaction. 
Next, radical trapping experiments were carried out, as shown in Scheme 7, and the addition of 2,2,6,6-tetramethyl-1-piperidinyloxy (TEMPO) drastically hampered the reaction. In addition, an EPR experiment was done (for detailed EPR spectra, see SI). Both results suggested that the radical mechanism might be involved in the reaction.
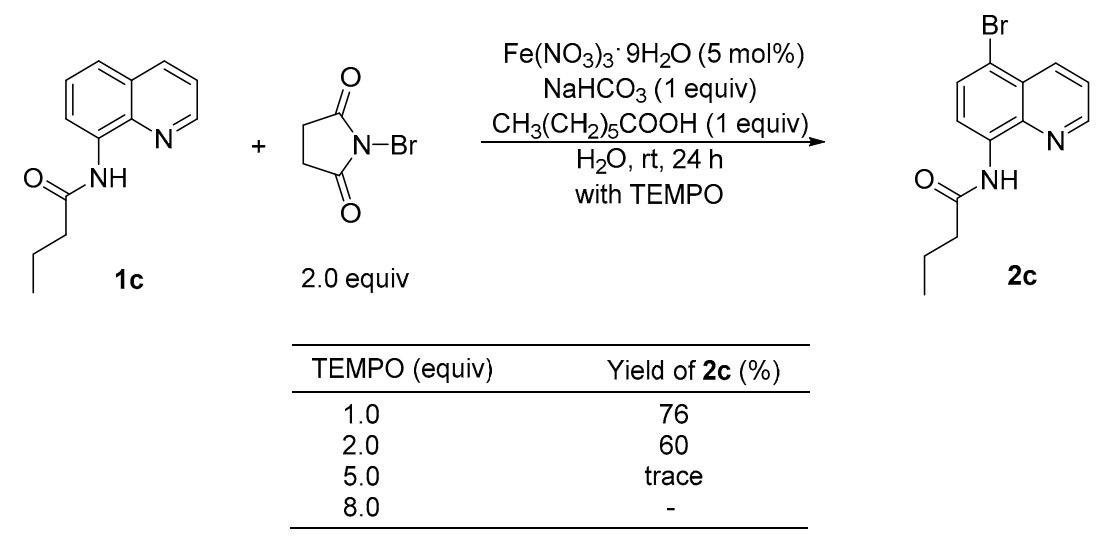

Scheme 7. Radical trapping experiments.

At last, three analogous substrates were also investigated. As shown in Scheme 8, 8 -aminoquinoline and quinoline gave $40 \%$ and none of the product, respectively, which suggested the necessity of the protected amino group during the reaction (Scheme 8a). Moreover, $N$-(1-naphthyl)carboxamide generated a mixture of brominated byproducts, indicating that a chelation of iron with $\mathrm{N}, \mathrm{N}$-bidentate 8 -aminoquinoline might play a predominant role in the reaction (Scheme $8 \mathrm{c}$ ).

a<smiles>Nc1cccc2cccnc12</smiles>

b<smiles>c1ccc2ncccc2c1</smiles>

c<smiles>CC(C)(C)C(=O)Nc1cccc2ccccc12</smiles>

standard conditions<smiles>CCC</smiles>

standard conditions<smiles></smiles><smiles>Nc1ccc(Br)c2cccnc12</smiles>

$40 \%$<smiles>Nc1c(Br)ccc2cccnc12</smiles>

$5 \%$<smiles>Nc1c(Br)cc(Br)c2cccnc12</smiles>

$3 \%$

$\stackrel{\text { standard conditions }}{\longrightarrow}$ No Brominated Product<smiles>CCCC(CBr)NC(=O)C(C)(C)C</smiles><smiles>CC(C)(C)C(=O)Nc1ccc(Br)c2ccccc12</smiles><smiles>CC(C)(C)C(=O)Nc1c(Br)cc(Br)c2ccccc12</smiles>

Scheme 8. The exploration of the analogous substrates.

Based on our work, as well as existing literatures [20-27], a plausible reaction pathway was proposed, as shown in Scheme 9. At first, substrate 1 and Fe(III) species formed complex A, which was transformed to be complex B after deprotonation [41,47]. Then, B, which may have influenced the electron density of the quinoline ring at the $\mathrm{C} 5-\mathrm{H}$ position $[47,48]$, was attacked by a bromine radical from the halogen reagent to form complex $C$ by a single electron transfer process (SET). The complex $C$ soon transformed into D through oxidation. After generation of the intermediate $\mathrm{E}$ through the proton transfer process (PT), a metal dissociation process gained the terminal product 2 and Fe(III) species, and the catalytic cycle was completed. Furthermore, considering that the reaction could be carried 
out without a catalyst, although the yield was low, a metal-free halogenation mechanism reported by $\mathrm{Xu}$ [32] also may have been involved in the reaction.

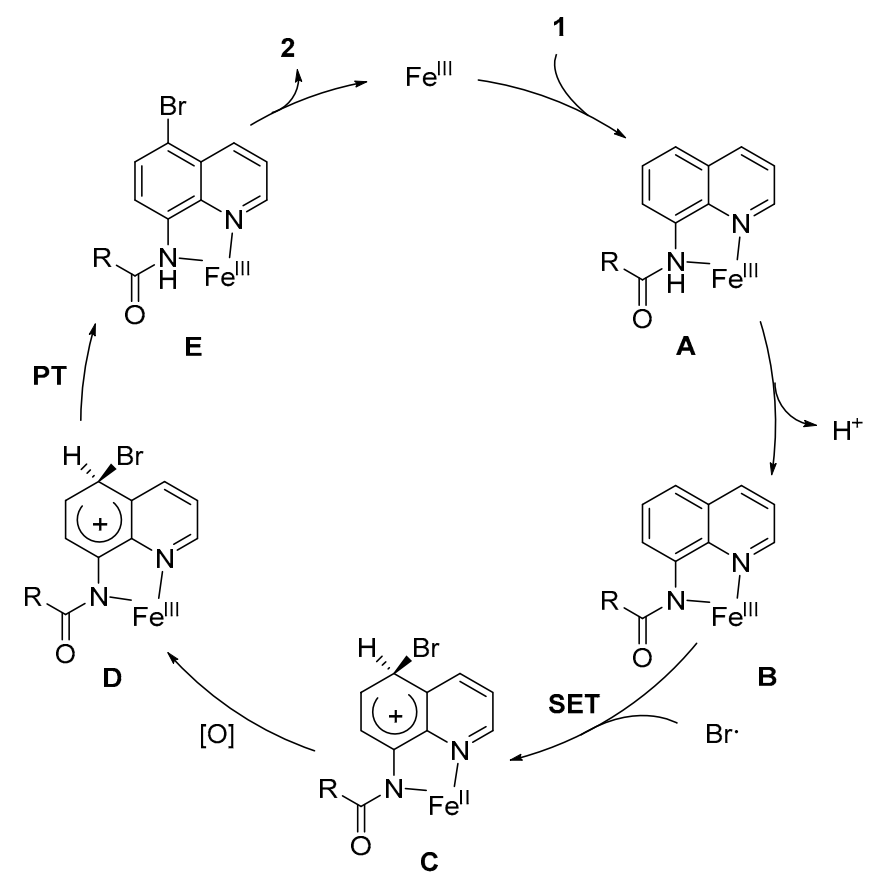

Scheme 9. Plausible mechanism for C5 halogenation of quinolines.

\section{Materials and Methods}

\subsection{General Experimental Procedures}

Unless otherwise noted, all the reactions were performed under air atmosphere. All reagents were used without purification as commercially available. All reactions were monitored by thin layer chromatography. Analytical thin layer chromatography (TLC) was performed using silica gel GF 254 plates. Chemical yields refer to pure isolated substances. Column chromatography was performed using silica gel (200-300 mesh or 300-400 mesh) eluting with petroleum ether and ethyl acetate. All products were characterized by their NMR spectra. ${ }^{1} \mathrm{H}-\mathrm{NMR}$ spectra were recorded at $400 \mathrm{MHz}$ and ${ }^{13} \mathrm{C}-\mathrm{NMR}$ spectra at $100 \mathrm{MHz}$ (Bruker DPX, Bruker, Madison, WI, USA) with $\mathrm{CDCl}_{3}$ as a solvent. Chemical shifts were reported in ppm using TMS as the internal standard.

\subsection{Synthesis of Starting Materials}

To a $50 \mathrm{~mL}$ single neck flask charged with $\mathrm{CH}_{2} \mathrm{Cl}_{2}(20 \mathrm{~mL})$ was added 8-aminoquinoline (10 mmol) and triethylamine $(11 \mathrm{mmol})$ and stirred at room temperature for $5 \mathrm{~min}$, then the reaction solution was cooled in an ice bath. The acid chloride $(12 \mathrm{mmol})$ was added dropwise (if solid, it was dissolved with $\mathrm{CH}_{2} \mathrm{Cl}_{2}$ ). The reaction solution was stirred overnight. When it was completed monitored by TLC, the mixture was filtered through a pad of Celite, the solid was washed with ethyl acetate $(30 \mathrm{~mL})$, and the organic layer was washed with $1 \mathrm{M} \mathrm{NaHCO}_{3}$ of aqueous solution $(3 \times 15 \mathrm{~mL})$, then the organic layer was dried with $\mathrm{Na}_{2} \mathrm{SO}_{4}$, filtered, and the solvent was removed under reduced pressure. The product was finally obtained by column chromatography on silica gel (PE/EtOAc $=20 / 1)$.

3.3. General Procedures for Iron-Catalyzed Halogenation C5-H of 8-Amidoquinolines under Mild Conditions in Water

Reaction conditions A: A mixture of 1 ( $0.3 \mathrm{mmol}), \mathrm{NBS}(0.6 \mathrm{mmol}), \mathrm{Fe}\left(\mathrm{NO}_{3}\right)_{3} \cdot 9 \mathrm{H}_{2} \mathrm{O}(5 \mathrm{~mol} \%)$, $\mathrm{CH}_{3}\left(\mathrm{CH}_{2}\right)_{5} \mathrm{COOH}(0.3 \mathrm{mmol}), \mathrm{NaHCO}_{3}(0.3 \mathrm{mmol})$ in water $(1.0 \mathrm{~mL})$ in a $20 \mathrm{~mL}$ Schlenk tube was stirred at room temperature for $24 \mathrm{~h}$. Then, the mixture was extracted with EtOAc 
$(10 \mathrm{~mL} \times 4)$. The combined organic layer was dried with $\mathrm{Na}_{2} \mathrm{SO}_{4}$ and filtered through a pad of Celite. The solvent was removed under reduced pressure and the residue was purified by silica gel column chromatography using PE/EtOAc (20/1) as an eluent to afford the products.

Reaction conditions $\mathrm{B}$ : A mixture of $1(0.3 \mathrm{mmol}), \mathrm{Br}_{2}(0.6 \mathrm{mmol}), \mathrm{Fe}\left(\mathrm{NO}_{3}\right)_{3} \cdot 9 \mathrm{H}_{2} \mathrm{O}(5 \mathrm{~mol} \%)$, $\mathrm{CH}_{3}\left(\mathrm{CH}_{2}\right)_{5} \mathrm{COOH}(0.3 \mathrm{mmol}), \mathrm{NaHCO}_{3}(0.3 \mathrm{mmol})$ in water $(1.0 \mathrm{~mL})$ in a $20 \mathrm{~mL}$ Schlenk tube was stirred at room temperature for $24 \mathrm{~h}$. Then, the mixture was extracted with EtOAc $(10 \mathrm{~mL} \times 4)$. The combined organic layer was dried with $\mathrm{Na}_{2} \mathrm{SO}_{4}$ and filtered through a pad of Celite. The solvent was removed under reduced pressure and the residue was purified by silica gel column chromatography using PE/EtOAc (20/1) as an eluent to afford the corresponding halogenation products.

Reaction conditions C: A mixture of $1(0.3 \mathrm{mmol}), \mathrm{NIS}(0.6 \mathrm{mmol}), \mathrm{Fe}\left(\mathrm{NO}_{3}\right)_{3} \cdot 9 \mathrm{H}_{2} \mathrm{O}(5 \mathrm{~mol} \%)$, $\mathrm{CH}_{3}\left(\mathrm{CH}_{2}\right)_{5} \mathrm{COOH}(0.3 \mathrm{mmol}), \mathrm{NaHCO}_{3}(0.3 \mathrm{mmol})$ in water $(1.0 \mathrm{~mL})$ in a $20 \mathrm{~mL}$ Schlenk tube was stirred at room temperature for $24 \mathrm{~h}$. Then, the mixture was extracted with EtOAc $(10 \mathrm{~mL} \times 4)$. The combined organic layer was dried with $\mathrm{Na}_{2} \mathrm{SO}_{4}$ and filtered through a pad of Celite. The solvent was removed under reduced pressure and the residue was purified by silica gel column chromatography using PE/EtOAc (20/1) as an eluent to afford the products.

Reaction conditions D: A mixture of $1(0.3 \mathrm{mmol}), \mathrm{I}_{2}(0.6 \mathrm{mmol}), \mathrm{Fe}\left(\mathrm{NO}_{3}\right)_{3} \cdot 9 \mathrm{H}_{2} \mathrm{O}(5 \mathrm{~mol} \%)$, $\mathrm{CH}_{3}\left(\mathrm{CH}_{2}\right)_{5} \mathrm{COOH}(0.3 \mathrm{mmol}), \mathrm{NaHCO}_{3}(0.3 \mathrm{mmol})$ in water $(1.0 \mathrm{~mL})$ in a $20 \mathrm{~mL}$ Schlenk tube was stirred at room temperature for $24 \mathrm{~h}$. Then, the mixture was extracted with EtOAc $(10 \mathrm{~mL} \times 4)$. The combined organic layer was dried with $\mathrm{Na}_{2} \mathrm{SO}_{4}$ and filtered through a pad of Celite. The solvent was removed under reduced pressure and the residue was purified by silica gel column chromatography using PE/EtOAc (20/1) as an eluent to afford the products.

$\mathrm{N}$-(5-Bromoquinolin-8-yl)acetamide (2a), ${ }^{1} \mathrm{H}-\mathrm{NMR}\left(400 \mathrm{MHz}, \mathrm{CDCl}_{3}\right) \delta=9.72(\mathrm{~s}, 1 \mathrm{H}), 8.77(\mathrm{dd}, J=4.2$, $1.5 \mathrm{~Hz}, 1 \mathrm{H}), 8.61(\mathrm{~d}, J=8.4 \mathrm{~Hz}, 1 \mathrm{H}), 8.46(\mathrm{dd}, J=8.5,1.6 \mathrm{~Hz}, 1 \mathrm{H}), 7.73(\mathrm{~d}, J=8.4 \mathrm{~Hz}, 1 \mathrm{H}), 7.51(\mathrm{dd}, J=8.5$, $4.2 \mathrm{~Hz}, 1 \mathrm{H}), 2.34(\mathrm{~s}, 3 \mathrm{H}) .{ }^{13} \mathrm{C}-\mathrm{NMR}\left(100 \mathrm{MHz}, \mathrm{CDCl}_{3}\right) \delta=168.78,148.55,138.81,135.90,134.37,130.85$, 127.05, 122.62, 116.82, 114.10, 25.18. HRMS: calculated $\left[\mathrm{C}_{11} \mathrm{H}_{10} \mathrm{BrN}_{2} \mathrm{O}\right]^{+}:$264.9971, found: 264.9964 [26].

$\mathrm{N}$-(5-Bromoquinolin-8-yl)propionamide (2b), ${ }^{1} \mathrm{H}-\mathrm{NMR}\left(400 \mathrm{MHz}, \mathrm{CDCl}_{3}\right) \delta=9.76(\mathrm{~s}, 1 \mathrm{H}), 8.78(\mathrm{dd}, J=4.2$, $1.6 \mathrm{~Hz}, 1 \mathrm{H}), 8.65(\mathrm{~d}, J=8.4 \mathrm{~Hz}, 1 \mathrm{H}), 8.47(\mathrm{dd}, J=8.5,1.6 \mathrm{~Hz}, 1 \mathrm{H}), 7.75(\mathrm{~d}, J=8.4 \mathrm{~Hz}, 1 \mathrm{H}), 7.52(\mathrm{dd}, J=8.5$, $4.2 \mathrm{~Hz}, 1 \mathrm{H}), 2.59(\mathrm{q}, J=7.6 \mathrm{~Hz}, 2 \mathrm{H}), 1.33(\mathrm{t}, J=7.6 \mathrm{~Hz}, 3 \mathrm{H}) .{ }^{13} \mathrm{C}-\mathrm{NMR}\left(100 \mathrm{MHz}, \mathrm{CDCl}_{3}\right) \delta=172.49$, $148.55,138.94,135.91,134.43,130.90,127.09,122.61,116.82,113.95,31.25,9.68$. HRMS: calculated $\left[\mathrm{C}_{12} \mathrm{H}_{12} \mathrm{BrN}_{2} \mathrm{O}\right]^{+}:$279.0128, found: 279.0122 [31].

$\mathrm{N}$-(5-Bromoquinolin-8-yl)butyramide (2c), ${ }^{1} \mathrm{H}-\mathrm{NMR}\left(400 \mathrm{MHz}, \mathrm{CDCl}_{3}\right) \delta=9.75(\mathrm{~s}, 1 \mathrm{H}), 8.78(\mathrm{dd}, J=4.2$, $1.5 \mathrm{~Hz}, 1 \mathrm{H}), 8.65(\mathrm{~d}, J=8.4 \mathrm{~Hz}, 1 \mathrm{H}), 8.47(\mathrm{dd}, J=8.5,1.5 \mathrm{~Hz}, 1 \mathrm{H}), 7.74(\mathrm{~d}, J=8.4 \mathrm{~Hz}, 1 \mathrm{H}), 7.52(\mathrm{dd}$, $J=8.5,4.2 \mathrm{~Hz}, 1 \mathrm{H}), 2.56-2.50(\mathrm{~m}, 2 \mathrm{H}), 1.90-1.78(\mathrm{~m}, 2 \mathrm{H}), 1.05(\mathrm{t}, J=7.4 \mathrm{~Hz}, 3 \mathrm{H}) .{ }^{13} \mathrm{C}-\mathrm{NMR}(100 \mathrm{MHz}$, $\left.\mathrm{CDCl}_{3}\right) \delta=171.75,148.50,138.87,135.92,134.38,130.88,127.06,122.58,116.84,113.94,40.11,19.05,13.83$. HRMS: calculated [ $\left.\mathrm{C}_{13} \mathrm{H}_{14} \mathrm{BrN}_{2} \mathrm{O}\right]^{+}$: 293.0284, found: 293.0284 [26].

$\mathrm{N}$-(5-Bromoquinolin-8-yl)hexanamide (2d), ${ }^{1} \mathrm{H}-\mathrm{NMR}\left(400 \mathrm{MHz}, \mathrm{CDCl}_{3}\right) \delta=9.74(\mathrm{~s}, 1 \mathrm{H}), 8.77(\mathrm{dd}, J=4.2$, $1.6 \mathrm{~Hz}, 1 \mathrm{H}), 8.65(\mathrm{~d}, J=8.4 \mathrm{~Hz}, 1 \mathrm{H}), 8.46(\mathrm{dd}, J=8.5,1.6 \mathrm{~Hz}, 1 \mathrm{H}), 7.74(\mathrm{~d}, J=8.4 \mathrm{~Hz}, 1 \mathrm{H}), 7.51(\mathrm{dd}$, $J=8.5,4.2 \mathrm{~Hz}, 1 \mathrm{H}), 2.62-2.44(\mathrm{~m}, 2 \mathrm{H}), 1.90-1.74(\mathrm{~m}, 2 \mathrm{H}), 1.45-1.32(\mathrm{~m}, 4 \mathrm{H}), 0.91(\mathrm{t}, J=7.1 \mathrm{~Hz}, 3 \mathrm{H})$. ${ }^{13} \mathrm{C}-\mathrm{NMR}\left(100 \mathrm{MHz}, \mathrm{CDCl}_{3}\right) \delta=171.89,148.52,138.89,135.83,134.42,130.85,127.03,122.57,116.79$, $113.92,38.19,31.45,25.27,22.48,13.99$. HRMS: calculated $\left[\mathrm{C}_{15} \mathrm{H}_{18} \mathrm{BrN}_{2} \mathrm{O}\right]^{+}: 321.0597$, found: 321.0587.

$N$-(5-Bromoquinolin-8-yl)isobutyramide (2e), ${ }^{1} \mathrm{H}-\mathrm{NMR}\left(400 \mathrm{MHz}, \mathrm{CDCl}_{3}\right) \delta=9.86(\mathrm{~s}, 1 \mathrm{H}), 8.80(\mathrm{~d}$, $J=4.2 \mathrm{~Hz}, 1 \mathrm{H}), 8.67(\mathrm{~d}, J=8.4 \mathrm{~Hz}, 1 \mathrm{H}), 8.48(\mathrm{~d}, J=8.5 \mathrm{~Hz}, 1 \mathrm{H}), 7.76(\mathrm{~d}, J=8.4 \mathrm{~Hz}, 1 \mathrm{H}), 7.53(\mathrm{dd}$, $J=8.5,4.2 \mathrm{~Hz}, 1 \mathrm{H}), 2.76(\mathrm{dt}, J=13.8,6.9 \mathrm{~Hz}, 1 \mathrm{H}), 1.35(\mathrm{~d}, J=6.9 \mathrm{~Hz}, 6 \mathrm{H}) .{ }^{13} \mathrm{C}-\mathrm{NMR}\left(100 \mathrm{MHz}, \mathrm{CDCl}_{3}\right)$ $\delta=175.73,148.56,139.07,135.92,134.49,130.89,127.09,122.58,116.86,113.93,37.12,19.66$. HRMS: calculated $\left[\mathrm{C}_{13} \mathrm{H}_{14} \mathrm{BrN}_{2} \mathrm{O}\right]^{+}: 293.0284$, found: 293.0284 [28]. 
N-(5-Bromoquinolin-8-yl)pivalamide (2f), ${ }^{1} \mathrm{H}-\mathrm{NMR}\left(400 \mathrm{MHz}, \mathrm{CDCl}_{3}\right) \delta=10.13(\mathrm{~s}, 1 \mathrm{H}), 8.71(\mathrm{dd}, J=4.2$, $1.6 \mathrm{~Hz}, 1 \mathrm{H}), 8.59(\mathrm{~d}, J=8.4 \mathrm{~Hz}, 1 \mathrm{H}), 8.37(\mathrm{dd}, J=8.5,1.6 \mathrm{~Hz}, 1 \mathrm{H}), 7.67(\mathrm{~d}, J=8.4 \mathrm{~Hz}, 1 \mathrm{H}), 7.43(\mathrm{dd}$, $J=8.5,4.2 \mathrm{~Hz}, 1 \mathrm{H}), 1.33(\mathrm{~s}, 9 \mathrm{H}) .{ }^{13} \mathrm{C}-\mathrm{NMR}\left(100 \mathrm{MHz}, \mathrm{CDCl}_{3}\right) \delta=176.19,147.60,138.34,134.79,133.53$, $129.83,126.02,121.50,115.62,112.82,39.32,26.64$. HRMS: calculated $\left[\mathrm{C}_{14} \mathrm{H}_{15} \mathrm{BrN}_{2} \mathrm{NaO}\right]^{+}$: 329.0260 , found: 329.0253 [26].

$\mathrm{N}$-(5-Bromoquinolin-8-yl)-2,2-dimethylbutanamide (2g), ${ }^{1} \mathrm{H}-\mathrm{NMR}\left(400 \mathrm{MHz}, \mathrm{CDCl}_{3}\right) \delta=10.10(\mathrm{~s}, 1 \mathrm{H}), 8.71$ $(\mathrm{d}, J=4.2 \mathrm{~Hz}, 1 \mathrm{H}), 8.60(\mathrm{~d}, J=8.4 \mathrm{~Hz}, 1 \mathrm{H}), 8.44-8.30(\mathrm{~m}, 1 \mathrm{H}), 7.67(\mathrm{~d}, J=8.4 \mathrm{~Hz}, 1 \mathrm{H}), 7.43(\mathrm{ddd}, J=8.5$, $4.2,0.7 \mathrm{~Hz}, 1 \mathrm{H}), 1.66(\mathrm{q}, J=7.5 \mathrm{~Hz}, 2 \mathrm{H}), 1.29(\mathrm{~s}, 6 \mathrm{H}), 0.85(\mathrm{t}, J=7.5 \mathrm{~Hz}, 3 \mathrm{H}) .{ }^{13} \mathrm{C}-\mathrm{NMR}\left(100 \mathrm{MHz}, \mathrm{CDCl}_{3}\right)$ $\delta=175.62,147.62,138.35,134.80,133.51,129.85,126.04,121.50,115.61,112.78,43.05,33.04,23.99,8.26$. HRMS: calculated $\left[\mathrm{C}_{15} \mathrm{H}_{18} \mathrm{BrN}_{2} \mathrm{O}\right]^{+}$: 321.0597 , found: 321.0587 .

$\mathrm{N}$-(5-Bromoquinolin-8-yl)-3-chloro-2,2-dimethylpropanamide (2h), ${ }^{1} \mathrm{H}-\mathrm{NMR}\left(400 \mathrm{MHz}, \mathrm{CDCl}_{3}\right) \delta=10.21$ (s, $1 \mathrm{H}), 8.71(\mathrm{dd}, J=4.2,1.6 \mathrm{~Hz}, 1 \mathrm{H}), 8.58(\mathrm{~d}, J=8.4 \mathrm{~Hz}, 1 \mathrm{H}), 8.36(\mathrm{dd}, J=8.5,1.6 \mathrm{~Hz}, 1 \mathrm{H}), 7.67(\mathrm{~d}, J=8.4 \mathrm{~Hz}$, $1 \mathrm{H}), 7.43(\mathrm{dd}, J=8.5,4.2 \mathrm{~Hz}, 1 \mathrm{H}), 3.69(\mathrm{~s}, 2 \mathrm{H}), 1.45(\mathrm{~s}, 6 \mathrm{H}) .{ }^{13} \mathrm{C}-\mathrm{NMR}\left(100 \mathrm{MHz}, \mathrm{CDCl}_{3}\right) \delta=172.42$, $147.77,138.28,134.81,132.99,129.74,126.02,121.62,115.89,113.44,51.65,44.79,22.61$. HRMS: calculated $\left[\mathrm{C}_{14} \mathrm{H}_{14} \mathrm{BrClN}_{2} \mathrm{NaO}\right]^{+}: 362.9870$, found: 362.9869 .

$\mathrm{N}$-(5-Bromoquinolin-8-yl)-3-cyclopentylpropanamide (2i), ${ }^{1} \mathrm{H}-\mathrm{NMR}\left(400 \mathrm{MHz}, \mathrm{CDCl}_{3}\right) \delta=9.76(\mathrm{~s}, 1 \mathrm{H})$, $8.84-8.76(\mathrm{~m}, 1 \mathrm{H}), 8.66(\mathrm{~d}, J=8.4 \mathrm{~Hz}, 1 \mathrm{H}), 8.48(\mathrm{dt}, J=8.5,1.4 \mathrm{~Hz}, 1 \mathrm{H}), 7.76(\mathrm{~d}, J=8.4 \mathrm{~Hz}, 1 \mathrm{H}), 7.53$ (ddd, $J=8.5,4.2,0.9 \mathrm{~Hz}, 1 \mathrm{H}), 2.61-2.53(\mathrm{~m}, 2 \mathrm{H}), 1.88-1.78(\mathrm{~m}, 5 \mathrm{H}), 1.69-1.47(\mathrm{~m}, 4 \mathrm{H}), 1.17(\mathrm{tt}, J=14.0$, $6.8 \mathrm{~Hz}, 2 \mathrm{H}) .{ }^{13} \mathrm{C}-\mathrm{NMR}\left(100 \mathrm{MHz}, \mathrm{CDCl}_{3}\right) \delta=172.02,148.55,138.93,135.90,134.46,130.90,127.08$, $122.59,116.84,113.94,39.74,37.55,32.55,31.77,25.20$. HRMS: calculated $\left[\mathrm{C}_{17} \mathrm{H}_{19} \mathrm{BrN}_{2} \mathrm{NaO}^{+}: 369.0573\right.$, found: 369.0566 .

N-(5-Bromoquinolin-8-yl)-4-methylpentanamide (2j), ${ }^{1} \mathrm{H}-\mathrm{NMR}\left(400 \mathrm{MHz}, \mathrm{CDCl}_{3}\right) \delta=9.66(\mathrm{~s}, 1 \mathrm{H}), 8.69$ $(\mathrm{dd}, J=4.2,1.4 \mathrm{~Hz}, 1 \mathrm{H}), 8.55(\mathrm{~d}, J=8.4 \mathrm{~Hz}, 1 \mathrm{H}), 8.37(\mathrm{dd}, J=8.5,1.5 \mathrm{~Hz}, 1 \mathrm{H}), 7.65(\mathrm{~d}, J=8.4 \mathrm{~Hz}, 1 \mathrm{H})$, $7.42(\mathrm{dd}, J=8.5,4.2 \mathrm{~Hz}, 1 \mathrm{H}), 2.54-2.40(\mathrm{~m}, 2 \mathrm{H}), 1.68-1.55(\mathrm{~m}, 3 \mathrm{H}), 0.88(\mathrm{~d}, J=6.4 \mathrm{~Hz}, 6 \mathrm{H}) .{ }^{13} \mathrm{C}-\mathrm{NMR}$ $\left(100 \mathrm{MHz}, \mathrm{CDCl}_{3}\right) \delta=172.06,148.54,138.91,135.91,134.44,130.90,127.07,122.59,116.85,113.94,36.24$, 34.34, 27.83, 22.41. HRMS: calculated $\left[\mathrm{C}_{15} \mathrm{H}_{18} \mathrm{BrN}_{2} \mathrm{O}\right]^{+}: 321.0597$, found: 321.0588 [32].

$\mathrm{N}$-(5-Bromoquinolin-8-yl)benzamide (2k), ${ }^{1} \mathrm{H}-\mathrm{NMR}\left(400 \mathrm{MHz}, \mathrm{CDCl}_{3}\right) \delta=10.65(\mathrm{~s}, 1 \mathrm{H}), 8.87-8.74(\mathrm{~m}, 2 \mathrm{H})$, $8.47(\mathrm{dd}, J=8.5,1.5 \mathrm{~Hz}, 1 \mathrm{H}), 8.10-8.00(\mathrm{~m}, 2 \mathrm{H}), 7.79(\mathrm{~d}, J=8.4 \mathrm{~Hz}, 1 \mathrm{H}), 7.61-7.49(\mathrm{~m}, 4 \mathrm{H}) .{ }^{13} \mathrm{C}-\mathrm{NMR}$ $\left(100 \mathrm{MHz}, \mathrm{CDCl}_{3}\right) \delta=165.48,148.78,139.42,136.16,134.86,134.51,132.07,131.05,128.88,127.33,122.79$, 117.13, 114.48. HRMS: calculated $\left[\mathrm{C}_{16} \mathrm{H}_{12} \mathrm{BrN}_{2} \mathrm{O}\right]^{+}: 327.0128$, found: 327.0120 [26].

$\mathrm{N}$-(5-Bromoquinolin-8-yl)-4-methylbenzamide (21), 1H-NMR (400 MHz, CDCl3) $\delta=10.62(\mathrm{~s}, 1 \mathrm{H}), 8.89-8.72$ $(\mathrm{m}, 2 \mathrm{H}), 8.46(\mathrm{dd}, J=8.5,1.5 \mathrm{~Hz}, 1 \mathrm{H}), 7.93(\mathrm{~d}, J=8.2 \mathrm{~Hz}, 2 \mathrm{H}), 7.78(\mathrm{~d}, J=8.4 \mathrm{~Hz}, 1 \mathrm{H}), 7.52(\mathrm{dd}, J=8.5$, $4.2 \mathrm{~Hz}, 1 \mathrm{H}), 7.31$ (d, $J=7.9 \mathrm{~Hz}, 2 \mathrm{H}), 2.43(\mathrm{~s}, 3 \mathrm{H}) .{ }^{13} \mathrm{C}-\mathrm{NMR}\left(100 \mathrm{MHz}, \mathrm{CDCl}_{3}\right) \delta=165.48,148.71$, 142.62, 139.41, 136.17, 134.62, 132.04, 131.08, 129.54, 127.34, 127.30, 122.74, 117.10, 114.29, 21.61. HRMS: calculated $\left[\mathrm{C}_{17} \mathrm{H}_{14} \mathrm{BrN}_{2} \mathrm{O}\right]^{+}: 341.0284$, found: 341.0284 [24].

$\mathrm{N}$-(5-Bromoquinolin-8-yl)-4-chlorobenzamide (2m), ${ }^{1} \mathrm{H}-\mathrm{NMR}\left(400 \mathrm{MHz}, \mathrm{CDCl}_{3}\right) \delta=10.63(\mathrm{~s}, 1 \mathrm{H}), 8.84(\mathrm{dd}$, $J=4.2,1.6 \mathrm{~Hz}, 1 \mathrm{H}), 8.76(\mathrm{~d}, J=8.4 \mathrm{~Hz}, 1 \mathrm{H}), 8.51(\mathrm{dd}, J=8.5,1.6 \mathrm{~Hz}, 1 \mathrm{H}), 7.98(\mathrm{~d}, J=8.6 \mathrm{~Hz}, 2 \mathrm{H}), 7.81(\mathrm{~d}$, $J=8.4 \mathrm{~Hz}, 1 \mathrm{H}), 7.57(\mathrm{dd}, J=8.5,4.2 \mathrm{~Hz}, 1 \mathrm{H}), 7.52-7.49(\mathrm{~m}, 2 \mathrm{H}) .{ }^{13} \mathrm{C}-\mathrm{NMR}\left(100 \mathrm{MHz}, \mathrm{CDCl}_{3}\right) \delta=164.33$, 148.82, 139.31, 138.35, 136.21, 134.22, 133.20, 131.00, 129.13, 128.73, 127.30, 122.83, 117.18, 114.72. HRMS: calculated $\left[\mathrm{C}_{16} \mathrm{H}_{11} \mathrm{BrClN}_{2} \mathrm{O}\right]^{+}: 360.9738$, found: 360.9725 [32].

$\mathrm{N}$-(5-Bromoquinolin-8-yl)-4-(trifluoromethyl)benzamide (2n), ${ }^{1} \mathrm{H}-\mathrm{NMR}\left(400 \mathrm{MHz}, \mathrm{CDCl}_{3}\right) \delta=10.70(\mathrm{~s}, 1 \mathrm{H})$, $8.83(\mathrm{dd}, J=4.2,1.5 \mathrm{~Hz}, 1 \mathrm{H}), 8.76(\mathrm{~d}, J=8.4 \mathrm{~Hz}, 1 \mathrm{H}), 8.51(\mathrm{dd}, J=8.5,1.5 \mathrm{~Hz}, 1 \mathrm{H}), 8.14(\mathrm{~d}, J=8.2 \mathrm{~Hz}$, $2 \mathrm{H}), 7.80(\mathrm{dd}, J=8.4,3.4 \mathrm{~Hz}, 3 \mathrm{H}), 7.57(\mathrm{dd}, J=8.5,4.2 \mathrm{~Hz}, 1 \mathrm{H}) . .{ }^{13} \mathrm{C}-\mathrm{NMR}\left(100 \mathrm{MHz}, \mathrm{CDCl}_{3}\right) \delta=163.92$, 148.91, 139.23, 138.00, 136.14, 133.98, 133.78, 130.91, 127.75, 127.24, 125.91 (q, J = 3.7 Hz), 122.89, 122.33, 117.20, 115.02. HRMS: calculated $\left[\mathrm{C}_{17} \mathrm{H}_{10} \mathrm{BrF}_{3} \mathrm{~N}_{2} \mathrm{NaO}\right]^{+}: 416.9821$, found: 416.9817 [31]. 
N-(5-Bromoquinolin-8-yl)-3-chlorobenzamide (2o), ${ }^{1} \mathrm{H}-\mathrm{NMR}\left(400 \mathrm{MHz}, \mathrm{CDCl}_{3}\right) \delta=10.66(\mathrm{~s}, 1 \mathrm{H}), 8.88$ $(\mathrm{dd}, J=4.2,1.6 \mathrm{~Hz}, 1 \mathrm{H}), 8.79(\mathrm{~d}, J=8.4 \mathrm{~Hz}, 1 \mathrm{H}), 8.55(\mathrm{dd}, J=8.5,1.6 \mathrm{~Hz}, 1 \mathrm{H}), 8.04(\mathrm{t}, J=1.8 \mathrm{~Hz}, 1 \mathrm{H})$, 7.96-7.89 (m, 1H), $7.85(\mathrm{~d}, J=8.4 \mathrm{~Hz}, 1 \mathrm{H}), 7.66-7.53(\mathrm{~m}, 2 \mathrm{H}), 7.49(\mathrm{t}, J=7.8 \mathrm{~Hz}, 1 \mathrm{H}) .{ }^{13} \mathrm{C}-\mathrm{NMR}(100 \mathrm{MHz}$, $\left.\mathrm{CDCl}_{3}\right) \delta=162.94,147.88,138.32,135.58,135.08,134.06,133.12,131.03,129.91,129.11,126.66,126.24$, 124.23, 121.82, 116.14, 113.80. HRMS: calculated $\left[\mathrm{C}_{16} \mathrm{H}_{10} \mathrm{BrClN}_{2} \mathrm{O}\right]^{+}: 360.9743$, found: 360.9726 [24].

$\mathrm{N}$-(5-Bromoquinolin-8-yl)-3-methoxylbenzamide (2p), ${ }^{1} \mathrm{H}-\mathrm{NMR}\left(400 \mathrm{MHz}, \mathrm{CDCl}_{3}\right) \delta=10.70(\mathrm{~s}, 1 \mathrm{H})$, 9.01-8.73 (m, 2H), $8.55(\mathrm{dd}, J=8.5,1.6 \mathrm{~Hz}, 1 \mathrm{H}), 7.85(\mathrm{~d}, J=8.4 \mathrm{~Hz}, 1 \mathrm{H}), 7.66-7.57(\mathrm{~m}, 3 \mathrm{H}), 7.46(\mathrm{t}$, $J=8.2 \mathrm{~Hz}, 1 \mathrm{H}), 7.13(\mathrm{ddd}, J=8.3,2.5,1.0 \mathrm{~Hz}, 1 \mathrm{H}), 3.92(\mathrm{~s}, 3 \mathrm{H}) .{ }^{13} \mathrm{C}-\mathrm{NMR}\left(100 \mathrm{MHz}, \mathrm{CDCl}_{3}\right) \delta=165.30$, 160.04, 148.84, 139.45, 136.35, 136.06, 134.50, 131.00, 129.86, 127.28, 122.79, 119.06, 118.19, 117.04, 114.50, 112.71, 55.55. HRMS: calculated $\left[\mathrm{C}_{17} \mathrm{H}_{14} \mathrm{BrN}_{2} \mathrm{O}_{2}\right]^{+}: 357.0239$, found: 357.0217 [24].

$\mathrm{N}$-(5-Bromoquinolin-8-yl)-3-phenylpropanamide (2q), ${ }^{1} \mathrm{H}-\mathrm{NMR}\left(400 \mathrm{MHz}, \mathrm{CDCl}_{3}\right) \delta=9.72(\mathrm{~s}, 1 \mathrm{H}), 8.74$ $(\mathrm{dd}, J=4.2,1.6 \mathrm{~Hz}, 1 \mathrm{H}), 8.65(\mathrm{~d}, J=8.4 \mathrm{~Hz}, 1 \mathrm{H}), 8.46(\mathrm{dd}, J=8.5,1.6 \mathrm{~Hz}, 1 \mathrm{H}), 7.75(\mathrm{~d}, J=8.4 \mathrm{~Hz}, 1 \mathrm{H})$, $7.50(\mathrm{dd}, J=8.5,4.2 \mathrm{~Hz}, 1 \mathrm{H}), 7.31-7.27(\mathrm{~m}, 4 \mathrm{H}), 7.24-7.17(\mathrm{~m}, 1 \mathrm{H}), 3.17-3.10(\mathrm{~m}, 2 \mathrm{H}), 2.87(\mathrm{dd}, J=8.7$, $7.0 \mathrm{~Hz}, 2 \mathrm{H}) .{ }^{13} \mathrm{C}-\mathrm{NMR}\left(100 \mathrm{MHz}, \mathrm{CDCl}_{3}\right) \delta=170.77,148.55,140.66,138.88,135.88,134.30,130.88$, $128.62,128.44,127.07,126.34,122.64,116.92,114.13,39.71,31.40$. HRMS: calculated $\left[\mathrm{C}_{18} \mathrm{H}_{15} \mathrm{BrN}_{2} \mathrm{NaO}\right]^{+}$: 377.0260, found: 377.0256 [22].

N-(5-Bromoquinolin-8-yl)thiophene-2-carboxamide (2r), ${ }^{1} \mathrm{H}-\mathrm{NMR}\left(400 \mathrm{MHz}, \mathrm{CDCl}_{3}\right) \delta=10.58(\mathrm{~s}, 1 \mathrm{H})$, $8.89(\mathrm{dd}, J=4.2,1.5 \mathrm{~Hz}, 1 \mathrm{H}), 8.75(\mathrm{~d}, J=8.4 \mathrm{~Hz}, 1 \mathrm{H}), 8.58(\mathrm{dd}, J=8.5,1.5 \mathrm{~Hz}, 1 \mathrm{H}), 7.86(\mathrm{dd}, J=4.8$, $3.7 \mathrm{~Hz}, 2 \mathrm{H}), 7.65-7.53(\mathrm{~m}, 2 \mathrm{H}), 7.20(\mathrm{dd}, J=4.9,3.8 \mathrm{~Hz}, 1 \mathrm{H}) .{ }^{13} \mathrm{C}-\mathrm{NMR}\left(100 \mathrm{MHz}, \mathrm{CDCl}_{3}\right) \delta=159.95$, $148.77,139.73,139.11,136.04,134.20,131.19,130.97,128.60,127.92,127.23,122.76,117.00,114.44$. HRMS: calculated [ $\left.\mathrm{C}_{14} \mathrm{H}_{10} \mathrm{BrN}_{2} \mathrm{OS}\right]^{+}: 332.9692$, found: 332.9691 [24].

N-(5-Bromo-2-methyl-8-quinolinyl)-pivalamide (2s), ${ }^{1} \mathrm{H}-\mathrm{NMR}\left(400 \mathrm{MHz}, \mathrm{CDCl}_{3}\right) \delta=10.31(\mathrm{~s}, 1 \mathrm{H}), 8.63(\mathrm{~d}$, $J=8.4 \mathrm{~Hz}, 1 \mathrm{H}), 8.35(\mathrm{~d}, J=8.6 \mathrm{~Hz}, 1 \mathrm{H}), 7.69(\mathrm{~d}, J=8.4 \mathrm{~Hz}, 1 \mathrm{H}), 7.39(\mathrm{~d}, J=8.6 \mathrm{~Hz}, 1 \mathrm{H}), 2.76(\mathrm{~s}, 3 \mathrm{H}), 1.42$ $(\mathrm{s}, 6 \mathrm{H}) .{ }^{13} \mathrm{C}-\mathrm{NMR}\left(100 \mathrm{MHz}, \mathrm{CDCl}_{3}\right) \delta=177.15,157.71,138.78,135.93,133.89,129.85,125.31,123.34$, 116.62, 113.85, 40.39, 27.64, 25.17. HRMS: calculated $\left[\mathrm{C}_{15} \mathrm{H}_{18} \mathrm{BrN}_{2} \mathrm{O}\right]^{+}: 321.0603$, found: 321.0601 [30].

$\mathrm{N}$-(5-Iodoquinolin-8-yl)butyramide (3c), ${ }^{1} \mathrm{H}-\mathrm{NMR}\left(400 \mathrm{MHz}, \mathrm{CDCl}_{3}\right) \delta=9.79(\mathrm{~s}, 1 \mathrm{H}), 8.77(\mathrm{dd}, J=4.2$, $1.5 \mathrm{~Hz}, 1 \mathrm{H}), 8.57(\mathrm{~d}, J=8.3 \mathrm{~Hz}, 1 \mathrm{H}), 8.37(\mathrm{dd}, J=8.5,1.5 \mathrm{~Hz}, 1 \mathrm{H}), 8.07(\mathrm{~d}, J=8.3 \mathrm{~Hz}, 1 \mathrm{H}), 7.53(\mathrm{dd}$, $J=8.5,4.2 \mathrm{~Hz}, 1 \mathrm{H}), 2.56-2.52(\mathrm{~m}, 2 \mathrm{H}), 1.85(\mathrm{dd}, J=14.9,7.4 \mathrm{~Hz}, 2 \mathrm{H}), 1.06(\mathrm{t}, J=7.4 \mathrm{~Hz}, 3 \mathrm{H}) .{ }^{13} \mathrm{C}-\mathrm{NMR}$ $\left(100 \mathrm{MHz}, \mathrm{CDCl}_{3}\right) \delta=171.79,148.65,140.67,138.88,138.25,135.42,129.51,123.10,117.76,89.06,40.17$, 19.06, 13.84. HRMS: calculated $\left[\mathrm{C}_{13} \mathrm{H}_{14} \mathrm{IN}_{2} \mathrm{O}\right]^{+}: 341.0145$, found: 341.0139 [26].

$\mathrm{N}$-(5-Iodoquinolin-8-yl)pivalamide (3f), ${ }^{1} \mathrm{H}-\mathrm{NMR}\left(400 \mathrm{MHz}, \mathrm{CDCl}_{3}\right) \delta=10.26(\mathrm{~s}, 1 \mathrm{H}), 8.76(\mathrm{dd}, J=4.2$, $1.5 \mathrm{~Hz}, 1 \mathrm{H}), 8.56(\mathrm{~d}, J=8.3 \mathrm{~Hz}, 1 \mathrm{H}), 8.33(\mathrm{dd}, J=8.5,1.6 \mathrm{~Hz}, 1 \mathrm{H}), 8.05(\mathrm{~d}, J=8.3 \mathrm{~Hz}, 1 \mathrm{H}), 7.51(\mathrm{dd}$, $J=8.5,4.2 \mathrm{~Hz}, 1 \mathrm{H}), 1.42(\mathrm{~s}, 9 \mathrm{H}) .{ }^{13} \mathrm{C}-\mathrm{NMR}\left(100 \mathrm{MHz}, \mathrm{CDCl}_{3}\right) \delta=176.27,147.72,139.59,138.26,137.19$, $134.49,128.43,122.03,116.52,87.94,39.36,26.63$. HRMS: calculated $\left[\mathrm{C}_{14} \mathrm{H}_{16} \mathrm{IN}_{2} \mathrm{O}\right]^{+}$: 355.0302 , found: $355.0292[31]$.

3.4. General Procedures for Suzuki Coupling Reaction of N-(5-Bromoquinolin-8-yl)pivalamide (4a as an Example)

A mixture of $2 \mathbf{f}(0.3 \mathrm{mmol})$, phenylboronic acid $(0.36 \mathrm{mmol}), \mathrm{PdCl}_{2}(5 \mathrm{~mol} \%), \mathrm{KOH}(0.6 \mathrm{mmol})$, in solvent (2-propanol/water $=1 / 1.5,1.0 \mathrm{~mL}$ ) in a $20 \mathrm{~mL}$ Schlenk tube was stirred at $80^{\circ} \mathrm{C}$ for $24 \mathrm{~h}$. Then, the mixture was extracted with EtOAc $(10 \mathrm{~mL} \times 4)$. The combined organic layer was dried with $\mathrm{Na}_{2} \mathrm{SO}_{4}$ and filtered through a pad of Celite. The solvent was removed under reduced pressure and the residue was purified by silica gel column chromatography using PE/EtOAc (20/1) as an eluent to afford the products.

$\mathrm{N}$-(5-Phenylquinolin-8-yl)pivalamide (4a), ${ }^{1} \mathrm{H}-\mathrm{NMR}\left(400 \mathrm{MHz}, \mathrm{CDCl}_{3}\right) \delta=10.38(\mathrm{~s}, 1 \mathrm{H}), 9.00-8.71(\mathrm{~m}, 2 \mathrm{H})$, $8.29(\mathrm{dd}, J=8.5,1.6 \mathrm{~Hz}, 1 \mathrm{H}), 7.54-7.39(\mathrm{~m}, 7 \mathrm{H}), 1.46(\mathrm{~s}, 9 \mathrm{H}) .{ }^{13} \mathrm{C}-\mathrm{NMR}\left(101 \mathrm{MHz}, \mathrm{CDCl}_{3}\right) \delta=177.34$, 
148.04, 139.27, 138.81, 134.76, 134.11, 134.03, 130.14, 128.52, 128.02, 127.45, 126.27, 121.51, 115.81, 40.42, 27.80. HRMS: calculated $\left[\mathrm{C}_{20} \mathrm{H}_{21} \mathrm{~N}_{2} \mathrm{O}\right]^{+}: 305.1648$, found: 305.1626 [30].

$\mathrm{N}$-(5-(4-Methoxyphenyl)quinolin-8-yl)pivalamide (4b), ${ }^{1} \mathrm{H}-\mathrm{NMR}\left(400 \mathrm{MHz}, \mathrm{CDCl}_{3}\right) \delta=10.35(\mathrm{~s}, 1 \mathrm{H}), 8.83$ $(\mathrm{dd}, J=4.8,3.2 \mathrm{~Hz}, 2 \mathrm{H}), 8.30(\mathrm{dd}, J=8.5,1.6 \mathrm{~Hz}, 1 \mathrm{H}), 7.49(\mathrm{~d}, J=8.0 \mathrm{~Hz}, 1 \mathrm{H}), 7.41(\mathrm{dd}, J=8.5$, $4.2 \mathrm{~Hz}, 1 \mathrm{H}), 7.39-7.35(\mathrm{~m}, 2 \mathrm{H}), 7.05-7.00(\mathrm{~m}, 2 \mathrm{H}), 3.89(\mathrm{~s}, 3 \mathrm{H}), 1.45(\mathrm{~s}, 9 \mathrm{H}) .{ }^{13} \mathrm{C}-\mathrm{NMR}\left(101 \mathrm{MHz}, \mathrm{CDCl}_{3}\right)$ $\delta=177.28,159.12,147.90,138.83,134.93,133.80,133.77,131.65,131.18,127.90,126.51,121.34,116.03$, 113.96, 55.41, 40.39, 27.80. HRMS: calculated $\left[\mathrm{C}_{21} \mathrm{H}_{23} \mathrm{~N}_{2} \mathrm{O}_{2}\right]^{+}: 355.1754$, found: 355.1736 [30].

$\mathrm{N}$-(5-(Pent-1-en-1-yl)quinolin-8-yl)pivalamide (4c), ${ }^{1} \mathrm{H}-\mathrm{NMR}\left(400 \mathrm{MHz}, \mathrm{CDCl}_{3}\right) \delta=10.30(\mathrm{~s}, 1 \mathrm{H}), 8.80$ $(\mathrm{dd}, J=4.2,1.6 \mathrm{~Hz}, 1 \mathrm{H}), 8.75(\mathrm{~d}, J=8.1 \mathrm{~Hz}, 1 \mathrm{H}), 8.47(\mathrm{dd}, J=8.6,1.6 \mathrm{~Hz}, 1 \mathrm{H}), 7.62(\mathrm{~d}, J=8.1 \mathrm{~Hz}, 1 \mathrm{H})$, $7.46(\mathrm{dd}, J=8.5,4.2 \mathrm{~Hz}, 1 \mathrm{H}), 6.96(\mathrm{~d}, J=15.6 \mathrm{~Hz}, 1 \mathrm{H}), 6.23(\mathrm{dt}, J=15.5,7.0 \mathrm{~Hz}, 1 \mathrm{H}), 2.29(\mathrm{~m}, J=7.2$, $1.5 \mathrm{~Hz}, 2 \mathrm{H}), 1.62-1.51(\mathrm{~m}, 2 \mathrm{H}), 1.43(\mathrm{~d}, J=1.6 \mathrm{~Hz}, 9 \mathrm{H}), 1.00(\mathrm{t}, J=7.4 \mathrm{~Hz}, 3 \mathrm{H}) .{ }^{13} \mathrm{C}-\mathrm{NMR}(100 \mathrm{MHz}$, $\left.\mathrm{CDCl}_{3}\right) \delta=177.14,147.84,138.72,134.26,133.70,132.79,129.66,125.77,125.26,124.41,121.12,116.21$, 40.35, 35.54, 27.77, 22.57, 13.81. HRMS: calculated $\left[\mathrm{C}_{19} \mathrm{H}_{25} \mathrm{~N}_{2} \mathrm{O}\right]^{+}: 297.1961$, found: 297.1950 [30].

$\mathrm{N}$-(5-(4-Chlorophenyl)quinolin-8-yl)pivalamide (4d), ${ }^{1} \mathrm{H}-\mathrm{NMR}\left(400 \mathrm{MHz}, \mathrm{CDCl}_{3}\right) \delta=10.36(\mathrm{~s}, 1 \mathrm{H})$, 8.96-8.71 (m, 2H), $8.23(\mathrm{dd}, J=8.5,1.6 \mathrm{~Hz}, 1 \mathrm{H}), 7.50-7.35(\mathrm{~m}, 6 \mathrm{H}), 1.45(\mathrm{~s}, 9 \mathrm{H}) .{ }^{13} \mathrm{C}-\mathrm{NMR}(101 \mathrm{MHz}$, $\left.\mathrm{CDCl}_{3}\right) \delta=177.37,148.08,138.76,137.69,134.46,134.42,133.58,132.64,131.38,128.72,128.10,126.16$, 121.63, 115.89, 40.42, 27.77. HRMS: calculated $\left[\mathrm{C}_{20} \mathrm{H}_{19} \mathrm{ClN}_{2} \mathrm{NaO}\right]^{+}: 361.1078$, found: 361.1069 [30].

$\mathrm{N}$-(5-(4-Cyanophenyl)quinolin-8-yl)pivalamide (4e), ${ }^{1} \mathrm{H}-\mathrm{NMR}\left(400 \mathrm{MHz}, \mathrm{CDCl}_{3}\right) \delta=10.38(\mathrm{~s}, 1 \mathrm{H}), 8.90-8.82$ (m, 2H), $8.19(\mathrm{dd}, J=8.6,1.5 \mathrm{~Hz}, 1 \mathrm{H}), 7.83-7.74(\mathrm{~m}, 2 \mathrm{H}), 7.61-7.55(\mathrm{~m}, 2 \mathrm{H}), 7.53-7.43(\mathrm{~m}, 2 \mathrm{H}), 1.45$ (s, 9H). ${ }^{13} \mathrm{C}-\mathrm{NMR}\left(101 \mathrm{MHz}, \mathrm{CDCl}_{3}\right) \delta=177.45,148.36,144.13,138.76,135.18,133.90,132.34,131.71$, $130.82,128.44,125.72,121.98,118.79,115.70,111.28,40.45,27.74$. HRMS: calculated $\left[\mathrm{C}_{21} \mathrm{H}_{19} \mathrm{~N}_{3} \mathrm{NaO}\right]^{+}$: 352.1420, found: 352.1411 [30].

\section{Conclusions}

In summary, we developed an efficient, economical, and environmentally friendly method for iron(III)-catalyzed C5-H halogenation of quinolines at room temperature in water. Both NXS and $\mathrm{X}_{2}$ could effectively function as halogen agents. The air could act as the oxidant. This transformation showed a broad substrate scope, good yield, and well-tolerated functionalization. Mechanism studies suggested that a single electron transfer (SET) mechanism might be involved in the reaction.

Supplementary Materials: The supplementary materials are available online.

Author Contributions: Y.L. and L.P. contributed equally. Y.L., L.P. and X.Z. conceived and designed the experiments; Y.L. and L.P. performed the experiments and analyzed the data. Y.L., L.P. and X.Z. wrote, read and approved the paper.

Funding: We are grateful to the National Natural Science Foundation of China (grant No. 21472128) and Sichuan Science and Technology Program (No. 2018JY560) for financial support.

Acknowledgments: We acknowledge Comprehensive Training Platform of Specialized Laboratory, College of Chemistry, Sichuan University for HRMS analysis.

Conflicts of Interest: The author declares no conflict of interest.

\section{References}

1. Snieckus, V. Directed ortho metalation. Tertiary amide and O-carbamate directors in synthetic strategies for polysubstituted aromatics. Chem. Rev. 1990, 90, 879-933. [CrossRef]

2. Shiraki, H.; Kozar, M.P.; Melendez, V.; Hudson, T.H.; Ohrt, C.; Magill, A.J.; Lin, A.J. Antimalarial Activity of Novel 5-Aryl-8-Aminoquinoline Derivatives. J. Med. Chem. 2011, 54, 131-142. [CrossRef] [PubMed]

3. Jain, M.; Vangapandu, S.; Sachdeva, S.; Jain, R. Synthesis and blood-schizontocidal antimalarial activities of 2-substituted /2,5-disubstituted-8-quinolinamines and some of their amino acid conjugates. Bioorganic Med. Chem. 2004, 12, 1003-1010. [CrossRef] [PubMed] 
4. Egan, T.J.; Ross, D.C.; Adams, P.A. Quinoline anti-malarial drugs inhibit spontaneous formation of $\beta$-haematin (malaria pigment). FEBS Lett. 1994, 352, 54-57. [CrossRef]

5. Foley, M.; Tilley, L. Quinoline Antimalarials: Mechanisms of Action and Resistance and Prospects for New Agents. Pharmacol. Ther. 1998, 79, 55-87. [CrossRef]

6. Hughes, G.; Bryce, M.R. Electron-transporting materials for organic electroluminescent and electrophosphorescent devices. J. Mater. Chem. 2005, 15, 94-107. [CrossRef]

7. Michael, J.P. Quinoline, quinazoline and acridone alkaloids. Nat. Prod. Rep. 2008, 25, 166-187. [CrossRef] [PubMed]

8. Solomon, V.R.; Lee, H. Quinoline as a Privileged Scaffold in Cancer Drug Discovery. Curr. Med. Chem. 2011, 18, 1488-1508. [CrossRef]

9. Zaitsev, V.G.; Shabashov, D.; Daugulis, O. Highly Regioselective Arylation of sp ${ }^{3}$ C-H Bonds Catalyzed by Palladium Acetate. J. Am. Chem. Soc. 2005, 127, 13154-13155. [CrossRef]

10. Rouquet, G.; Chatani, N. Catalytic Founctionalization of $\mathrm{C}\left(\mathrm{sp}^{2}\right)-\mathrm{H}$ and $\mathrm{C}\left(\mathrm{sp}^{3}\right)-\mathrm{H}$ Bonds by Using Bidentate Directing Groups. Angew. Chem. Int. Ed. 2013, 52, 11726-11743. [CrossRef]

11. Zhu, C.; Yi, M.; Wei, D.; Chen, X.; Wu, Y.; Cui, X. Copper-Catalyzed Direct Amination of Quinoline N-Oxides via C-H Bond Activation under Mild Conditions. Org. Lett. 2014, 16, 1840-1843. [CrossRef] [PubMed]

12. Chen, X.; Zhu, C.; Cui, X.; Wu, Y. Direct 2-acetoxylation of quinoline $\mathrm{N}$-oxides via copper catalyzed C-H bond activation. Chem. Commun. 2013, 49, 6900-6902. [CrossRef] [PubMed]

13. Wang, H.; Cui, X.; Pei, Y.; Zhang, Q.; Bai, J.; Wei, D.; Wu, Y. Direct regioselective phosphonation of heteroaryl $\mathrm{N}$-oxides with $\mathrm{H}$-phosphonates under metal and external oxidant free conditions. Chem. Commun. 2014, 50, 14409-14411. [CrossRef]

14. Li, G.; Jia, C.; Sun, K. Copper-Catalyzed Intermolecular Dehydrogenative Amidation/Amination of Quinoline N-Oxides with Lactams/Cyclamines. Org. Lett. 2013, 15, 5198-5201. [CrossRef] [PubMed]

15. Campeau, L.C.; Stuart, D.R.; Leclerc, J.P.; Bertrand-Laperle, M.; Villemure, E.; Sun, H.Y.; Lasserre, S.; Guimond, N.; Lecavallier, M.; Fagnou, K. Palladium-Catalyzed Direct Arylation of Azine and Azole N-Oxides: Reaction Development, Scope and Applications in Synthesis. J. Am. Chem. Soc. 2009, 131, 3291-3306. [CrossRef] [PubMed]

16. Sun, K.; Lv, Y.; Wang, J.; Sun, J.; Liu, L.; Jia, M.; Liu, X.; Li, Z.; Wang, X. Regioselective, Molecular Iodine-Mediated C3 Iodination of Quinolines. Org. Lett. 2015, 17, 4408-4411. [CrossRef] [PubMed]

17. Zhang, X.Y.; Qi, Z.S.; Li, X.W. Rhodium(III)-Catalyzed C-C and C-O Coupling of Quinoline N-Oxides with Alkynes: Combination of C-H Activation with O-Atom Transfer. Angew. Chem. Int. Ed. 2014, 53, 10794-10798. [CrossRef]

18. Jeong, J.; Patel, P.; Hwang, H.; Chang, S. Rhodium(III)-Catalyzed C-C Bond Formation of Quinoline N-Oxides at the C-8 Position under Mild Conditions. Org. Lett. 2014, 16, 4598-4601. [CrossRef]

19. Hwang, H.; Kim, J.; Jeong, J.; Chang, S. Regioselective Introduction of Heteroatoms at the C-8 Position of Quinoline N-Oxides: Remote C-H Activation Using N-Oxide as a Stepping Stone. J. Am. Chem. Soc. 2014, 136, 10770-10776. [CrossRef]

20. Suess, A.M.; Ertem, M.Z.; Cramer, C.J.; Stahl, S.S. Divergence between Organometallic and Single-Electron-Transfer Mechanisms in Copper(II)-Mediated Aerobic C-H Oxidation. J. Am. Chem. Soc. 2013, 135, 9797-9804. [CrossRef]

21. Guo, H.; Chen, M.; Jiang, P.; Chen, J.; Pan, L.; Wang, M.; Xie, C.; Zhang, Y. Copper and palladium mediated $\mathrm{C}-\mathrm{H}$ chlorination on 8-acylaminoquinoline scaffolds. Tetrahedron 2015, 71, 70-76. [CrossRef]

22. Xu, J.; Zhu, X.; Zhou, G.; Ying, B.; Ye, P.; Su, L.; Shen, C.; Zhang, P. Copper(II)-catalyzed C5 and C7 halogenation of quinolines using sodium halides under mild conditions. Org. Biomol. Chem. 2016, 14, 3016-3021. [CrossRef] [PubMed]

23. Ding, J.; Li, W.; Ye, K.; Li, J. The Highly Regioselective Halogenation of N-(8-quinolinyl)amides on the C-5 Position with Cuprous Halides Under Mild Conditions. ChemistrySelect 2016, 1, 5874-5878. [CrossRef]

24. Liu, X.X.; Wu, Z.Y.; Luo, X.L.; He, Y.Q.; Zhou, X.Q.; Fan, Y.X.; Huang, G.S. PhI(OAc) 2 oxidative C5 halogenation of quinolines using copper halides under mild conditions. RSC Adv. 2016, 6, 71485-71488. [CrossRef]

25. Wu, C.; Zhou, H.; Wu, Q.; He, M.; Li, P.; Su, Q.; Mu, Y. Copper-Catalyzed Regioselective C-H Iodination of Aromatic Carboxamides. Synlett 2016, 27, 868-875. [CrossRef] 
26. He, X.; Xu, Y.Z.; Kong, L.X.; Wu, H.H.; Ji, D.Z.; Wang, Z.B.; Xu, Y.G.; Zhu, Q.H. Copper(I) and $N$-fluorobenzenesulfonimide-mediated direct regioselective halogenation of 8-amidoquinolines on the C5 position. Org. Chem. Front. 2017, 4, 1046-1050. [CrossRef]

27. Rao, N.S.; Reddy, G.M.; Sridhar, B.; Sarma, M.H. Copper-Mediated Remote Highly Site-Selective C-H Bond Bromination and Chlorination of Quinolines at the C5 Position that is Geometrically Difficult to Access. Eur. J. Org. Chem. 2017, 438-442. [CrossRef]

28. Wang, Y.; Wang, Y.; Jiang, K.; Zhang, Q.; Li, D. Transition-metal-free oxidative C5 C-H-halogenation of 8-aminoquinoline amides using sodium halides. Org. Biomol. Chem. 2016, 14, 10180-10184. [CrossRef]

29. Jiao, J.Y.; Mao, Y.J.; Feng, A.W.; Li, X.F.; Li, M.T.; Zhang, X.H. The regioselective C5 halogenation of quinolines using sodium halides under transition metal-free conditions. Tetrahedron 2017, 73, 1482-1488. [CrossRef]

30. Li, Y.; Zhu, L.; Cao, X.; Au, C.T.; Qiu, R.; Yin, S.F. Metal-free C5-H Bromination of Quinolines for One-pot C-X (X=C, O, S) Bond Formations. Adv. Synth. Catal. 2017, 359, 2864-2873. [CrossRef]

31. Motati, D.R.; Uredi, D.; Watkins, E.B. A general method for the metal-free, regioselective, remote C-H halogenation of 8-substituted quinolines. Chem. Sci. 2018, 9, 1782-1788. [CrossRef] [PubMed]

32. Chen, J.; Wang, T.; Liu, Y.; Wang, T.; Lin, A.; Yao, H.; Xu, J. Metal-free C5-selective halogenation of quinolines under aqueous conditions. Org. Chem. Front. 2017, 4, 622-626. [CrossRef]

33. Liang, L.; Li, Z.; Zhou, X. Pyridine N-Oxides as Ligands in Cu-Catalyzed N-Arylation of Imidazoles in Water. Org. Lett. 2009, 11, 3294-3297. [CrossRef] [PubMed]

34. Ke, F.; Qu, Y.; Jiang, Z.; Li, Z.; Wu, D.; Zhou, X. An Efficient Copper-Catalyzed Carbon-Sulfur Bond Formation Protocol in Water. Org. Lett. 2011, 13, 454-457. [CrossRef]

35. Li, Z.; Ke, F.; Deng, H.; Xu, H.; Xiang, H.; Zhou, X. Synthesis of disulfides and diselenides by copper-catalyzed coupling reactions in water. Org. Biomol. Chem. 2013, 11, 2943-2946. [CrossRef] [PubMed]

36. Wu, Z.; Luo, F.; Chen, S.; Li, Z.; Xiang, H.; Zhou, X. Palladium-catalyzed para-selective arylation of phenols with aryl iodides in water. Chem. Commun. 2013, 49, 7653-7655. [CrossRef] [PubMed]

37. Pan, L.; Yu, L.; Wu, Z.; Li, Z.; Xiang, H.; Zhou, X. Quaternary ammonium salt as alkylation agent in three-component reactions for the synthesis of benzothiazoles in water. RSC Adv. 2014, 4, 27775-27779. [CrossRef]

38. Qu, Y.; Ke, F.; Zhou, L.; Li, Z.; Xiang, H.; Wu, D.; Zhou, X. Synthesis of 3-indole derivatives by copper sulfonato Salen catalyzed three-component reactions in water. Chem. Commun. 2011, 47, 3912-3914. [CrossRef]

39. Jing, L.; Wei, J.; Zhou, L.; Huang, Y.; Li, Z.; Zhou, X. Lithium pipecolinate as a facile and efficient ligand for copper-catalyzed hydroxylation of aryl halides in water. Chem. Commun. 2010, 46, 4767-4769. [CrossRef]

40. Yao, X.; Weng, X.; Wang, K.; Xiang, H.; Zhou, X. Transition metal free oxygenation of 8-aminoquinoline amides in water. Green Chem. 2018, 20, 2472-2476. [CrossRef]

41. Qiao, H.; Sun, S.; Yang, F.; Zhu, Y.; Kang, J.; Wu, Y.; Wu, Y. Merging Photoredox Catalysis with Iron(III) Catalysis: C5-H Bromination and Iodination of 8-Aminoquinoline Amides in Water. Adv. Synth. Catal. 2017, 359, 1976-1980. [CrossRef]

42. Hashimoto, T.; Maruoka, K. Recent development and application of chiral phase-transfer catalysts. Chem. Rev. 2007, 107, 5656-5682. [CrossRef] [PubMed]

43. Merkushev, E.B. Advances in the Synthesis of Iodoaromatic Compounds. Synthesis 1988, 12, $923-937$. [CrossRef]

44. Prakash, G.K.S.; Mathew, T.; Hoole, D.; Esteves, P.M.; Wang, Q.; Rasul, G.; Olah, G.A. $\mathrm{N}$-Halosuccinimide $/ \mathrm{BF}_{3}-\mathrm{H}_{2} \mathrm{O}$, Efficient Electrophilic Halogenating Systems for Aromatics. J. Am. Chem. Soc. 2004, 126, 15770-15776. [CrossRef] [PubMed]

45. Zawartka, W.; Póspiech, P.; Cypryk, M.; Trzeciak, A.M. Palladium supported on aminopropyl-functionalized polymethylsiloxane microspheres: Simple and effective catalyst for the Suzuki-Miyaura C-C coupling. J. Mol. Catal. A Chem. 2015, 407, 230-235. [CrossRef]

46. Mondal, M.; Bora, U. An efficient protocol for palladium-catalyzed ligand-free Suzuki-Miyaura coupling in water. Green Chem. 2012, 14, 1873-1876. [CrossRef]

47. Cui, M.; Liu, J.; Lu, X.; Lu, X.; Zhang, Z.; Xiao, B.; Fu, Y. Iron-mediated remote C-H bond benzylation of 8-aminoquinoline amides. Tetrahedron Lett. 2017, 58, 1912-1916. [CrossRef] 
48. Liang, H.; Jiang, K.; Ding, W.; Yuan, Y.; Shuai, L.; Chen, Y.; Wei, Y. Selective remote C-H sulfonylation of aminoquinolines with arylsulfonyl chlorides via copper catalysis. Chem. Commun. 2015, 51, 16928-16931. [CrossRef] [PubMed]

Sample Availability: Samples of the compounds 1-4 are available from the authors.

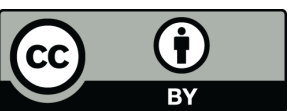

(C) 2019 by the authors. Licensee MDPI, Basel, Switzerland. This article is an open access article distributed under the terms and conditions of the Creative Commons Attribution (CC BY) license (http://creativecommons.org/licenses/by/4.0/). 